\title{
Powers of Octonions
}

\section{W. E. Ahmed1,2}

${ }^{1}$ Mathematics and Statistics Department, Faculty of Science, Imam Mohammad Ibn Saud Islamic University, Riyadh, KSA ${ }^{2}$ Department of Basics and Engineering Sciences, Faculty of Engineering, University of Khartoum, Khartoum, Sudan Email: waahmed@imamu.edu.sa

How to cite this paper: Ahmed, W.E. (2021) Powers of Octonions. Applied Mathematics, 12, 75-84

https://doi.org/10.4236/am.2021.122006

Received: December 27, 2020

Accepted: February 7, 2021

Published: February 10, 2021

Copyright (c) 2021 by author(s) and Scientific Research Publishing Inc. This work is licensed under the Creative Commons Attribution International License (CC BY 4.0).

http://creativecommons.org/licenses/by/4.0/

\begin{abstract}
As it is known, Binomial expansion, De Moivre's formula, and Euler's formula are suitable methods for computing the powers of a complex number, but to compute the powers of an octonion number in easy way, we need to derive suitable formulas from these methods. In this paper, we present a novel way to compute the powers of an octonion number using formulas derived from the binomial expansion.
\end{abstract}

\section{Keywords}

Octonion, Matrix Algebra

\section{Introduction}

An octonion number $a$ can be expressed as:

$$
a=a_{0}+a_{1} i_{1}+a_{2} i_{2}+a_{3} i_{3}+a_{4} i_{4}+a_{5} i_{5}+a_{6} i_{6}+a_{7} i_{7}
$$

where $a_{0}, \cdots, a_{7}$ are real numbers and $i_{1}, \cdots, i_{7}$ are imaginary units. Their multiplication is given in Table 1. Octonion algebra is an eight-dimensional, non-commutative, non-associative and normed division algebra.

Table 1. Imaginary units multiplication.

\begin{tabular}{cccccccc}
\hline & $i_{1}$ & $i_{2}$ & $i_{3}$ & $i_{4}$ & $i_{5}$ & $i_{6}$ & $i_{7}$ \\
\hline$i_{1}$ & -1 & $i_{3}$ & $-i_{2}$ & $i_{5}$ & $-i_{4}$ & $-i_{7}$ & $i_{6}$ \\
$i_{2}$ & $-i_{3}$ & -1 & $i_{1}$ & $i_{6}$ & $i_{7}$ & $-i_{4}$ & $-i_{5}$ \\
$i_{3}$ & $i_{2}$ & $-i_{1}$ & -1 & $i_{7}$ & $-i_{6}$ & $i_{5}$ & $-i_{4}$ \\
$i_{4}$ & $-i_{5}$ & $-i_{6}$ & $-i_{7}$ & -1 & $i_{1}$ & $i_{2}$ & $i_{3}$ \\
$i_{5}$ & $i_{4}$ & $-i_{7}$ & $i_{6}$ & $-i_{1}$ & -1 & $-i_{3}$ & $i_{2}$ \\
$i_{6}$ & $i_{7}$ & $i_{4}$ & $-i_{5}$ & $-i_{2}$ & $i_{3}$ & -1 & $-i_{1}$ \\
$i_{7}$ & $-i_{6}$ & $i_{5}$ & $i_{4}$ & $-i_{3}$ & $-i_{2}$ & $i_{1}$ & -1 \\
\hline
\end{tabular}


Octonions have been used in many fields of mathematics, and they have many applications, about octonions and their applications see [1] [2] [3], and [4] [5] to take a historical overview.

In the matrix representation, an octonion $a$ can be represented by $8 \times 8$ real matrices. One form of these matrices is a matrix $A$ (the left matrix representation) [6]:

$$
A=\left[\begin{array}{cccccccc}
a_{0} & -a_{1} & -a_{2} & -a_{3} & -a_{4} & -a_{5} & -a_{6} & -a_{7} \\
a_{1} & a_{0} & -a_{3} & a_{2} & -a_{5} & a_{4} & a_{7} & -a_{6} \\
a_{2} & a_{3} & a_{0} & -a_{1} & -a_{6} & -a_{7} & a_{4} & a_{5} \\
a_{3} & -a_{2} & a_{1} & a_{0} & -a_{7} & a_{6} & -a_{5} & a_{4} \\
a_{4} & a_{5} & a_{6} & a_{7} & a_{0} & -a_{1} & -a_{2} & -a_{3} \\
a_{5} & -a_{4} & a_{7} & -a_{6} & a_{1} & a_{0} & a_{3} & -a_{2} \\
a_{6} & -a_{7} & -a_{4} & a_{5} & a_{2} & -a_{3} & a_{0} & a_{1} \\
a_{7} & a_{6} & -a_{5} & -a_{4} & a_{3} & a_{2} & -a_{1} & a_{0}
\end{array}\right]
$$

The problem of computing the $\mathrm{n}^{\text {th }}$ power of an octonion is still interesting to many researchers. Some methods are used, such as binomial expansion, De Moivre's formula, and Euler's formula. To solve this problem, we use a new technique to construct formulas computing the powers of an octonion.

\section{The Methodology}

For a complex number $b=b_{0}+b_{1} i$ where $b_{0}, b_{1}$ are real numbers, and $i$ is the imaginary unit satisfies $i^{2}=-1$,

$$
b^{n}=\left(b_{0}+b_{1} i\right)^{n}=\sum_{j=0}^{n}\left(\begin{array}{c}
n \\
n-j
\end{array}\right)\left(b_{0}^{n-j}\right)\left(b_{1} i\right)^{j}, n \text { is a positive integer number }
$$

If $n$ is an even number then there will be $\frac{n}{2}+1$ real terms and $\frac{n}{2}$ imaginary terms, to simplify, we define $\operatorname{Im}[b]=b_{1} i, \operatorname{Re}[b]=b_{0}$ and, let $b_{0}, b_{1}$ be nonzero real numbers, we can write (2) as:

$$
\begin{aligned}
& \operatorname{Re}\left[b^{n}\right]=\sum_{j=0}^{n / 2}\left(\begin{array}{c}
n \\
n-2 j
\end{array}\right) b_{0}^{n-2 j}\left(-b_{1}^{2}\right)^{j} \\
& \operatorname{Im}\left[b^{n}\right]=\left(b-b_{0}\right) \sum_{j=0}^{n / 2-1}\left(\begin{array}{c}
n \\
n-2 j-1
\end{array}\right) b_{0}^{n-2 j-1}\left(-b_{1}^{2}\right)^{j}
\end{aligned}
$$

If $n$ is an odd number then there will be $\frac{n+1}{2}$ real terms and $\frac{n+1}{2}$ imaginary terms, we can write (2) as:

$$
\begin{aligned}
& \operatorname{Re}\left[b^{n}\right]=\sum_{j=0}^{(n-1) / 2}\left(\begin{array}{c}
n \\
n-2 j
\end{array}\right) b_{0}^{n-2 j}\left(-b_{1}^{2}\right)^{j} \\
& \operatorname{Im}\left[b^{n}\right]=\left(b-b_{0}\right) \sum_{j=0}^{(n-1) / 2}\left(\begin{array}{c}
n \\
n-2 j-1
\end{array}\right) b_{0}^{n-2 j-1}\left(-b_{1}^{2}\right)^{j}
\end{aligned}
$$

To prove (3), let us write $b$ in a matrix representation form: 


$$
B=\left[\begin{array}{cc}
b_{0} & -b_{1} \\
b_{1} & b_{0}
\end{array}\right]
$$

For $n=2, b^{2}$ can be computed from:

$$
\left[\begin{array}{cc}
b_{0} & -b_{1} \\
b_{1} & b_{0}
\end{array}\right]\left[\begin{array}{l}
b_{0} \\
b_{1}
\end{array}\right]=\left[\begin{array}{c}
b_{0}^{2}-b_{1}^{2} \\
2 b_{0} b_{1}
\end{array}\right],
$$

so

$$
\begin{aligned}
& \operatorname{Re}\left[b^{2}\right]=b_{0}^{2}-b_{1}^{2}=\sum_{j=0}^{1}\left(\begin{array}{c}
2 \\
2-2 j
\end{array}\right) b_{0}^{2-2 j}\left(-b_{1}^{2}\right)^{j} \\
& \operatorname{Im}\left[b^{2}\right]=2 b_{0} b_{1}=\left(b-b_{0}\right) \sum_{j=0}^{0}\left(\begin{array}{c}
2 \\
1-2 j
\end{array}\right) b_{0}^{1-2 j}\left(-b_{1}^{2}\right)^{j}
\end{aligned}
$$

Assume that (3) is true for $n=2 k$, where $k$ is a positive integer, therefore:

$$
\begin{aligned}
& \operatorname{Re}\left[b^{2 k}\right]=\sum_{j=0}^{k}\left(\begin{array}{c}
2 k \\
2 k-2 j
\end{array}\right) b_{0}^{2 k-2 j}\left(-b_{1}^{2}\right)^{j} \\
& \operatorname{Im}\left[b^{2 k}\right]=\left(b-b_{0}\right) \sum_{j=0}^{k-1}\left(\begin{array}{c}
2 k \\
2 k-2 j-1
\end{array}\right) b_{0}^{2 k-2 j-1}\left(-b_{1}^{2}\right)^{j}
\end{aligned}
$$

We can compute $b^{2 k+2}=b^{2} b^{2 k}$ by using the matrix:

$$
B^{2}=\left[\begin{array}{cc}
b_{0}^{2}-b_{1}^{2} & -2 b_{0} b_{1} \\
2 b_{0} b_{1} & b_{0}^{2}-b_{1}^{2}
\end{array}\right]
$$

Multiplying the first row of $B^{2}$ by $\left(b^{2 k}\right)^{\mathrm{T}}$ (the column matrix representing $\left.\left(b^{2 k}\right)\right)$ gives $\operatorname{Re}\left[b^{2 k+2}\right]$, and multiplying the second row of $B^{2}$ by $\left(b^{2 k}\right)^{\mathrm{T}}$ gives $\operatorname{Im}\left[b^{2 k+2}\right]$.

$$
\begin{aligned}
\operatorname{Re} & {\left[b^{2 k+2}\right]=\left(b_{0}^{2}-b_{1}^{2}\right) \sum_{j=0}^{k}\left(\begin{array}{c}
2 k \\
2 k-2 j
\end{array}\right) b_{0}^{2 k-2 j}\left(-b_{1}^{2}\right)^{j} } \\
& -2 b_{0} b_{1} \sum_{j=0}^{k-1}\left(\begin{array}{c}
2 k \\
2 k-2 j-1
\end{array}\right) b_{0}^{2 k-2 j-1}\left(-b_{1}^{2}\right)^{j} \\
= & \sum_{j=0}^{k}\left(\begin{array}{c}
2 k \\
2 k-2 j
\end{array}\right) b_{0}^{2 k-2 j+2}\left(-b_{1}^{2}\right)^{j}+\sum_{j=0}^{k}\left(\begin{array}{c}
2 k \\
2 k-2 j
\end{array}\right) b_{0}^{2 k-2 j}\left(-b_{1}^{2}\right)^{j+1} \\
& +2 \sum_{j=0}^{k-1}\left(\begin{array}{c}
2 k \\
2 k-2 j-1
\end{array}\right) b_{0}^{2 k-2 j}\left(-b_{1}^{2}\right)^{j+1} \\
= & b_{0}^{2 k+2}-\left(\begin{array}{c}
2 k \\
2 k-2
\end{array}\right) b_{0}^{2 k}\left(b_{1}^{2}\right)+\left(\begin{array}{c}
2 k \\
2 k-4
\end{array}\right) b_{0}^{2 k-2}\left(b_{1}^{2}\right)^{2}+\cdots \\
& +\left(\begin{array}{c}
2 k \\
2 k-2 r+2
\end{array}\right) b_{0}^{2 k-2 r+4}\left(-b_{1}^{2}\right)^{r-1}+\left(\begin{array}{c}
2 k \\
k-2 r
\end{array}\right) b_{0}^{2 k-2 r+2}\left(-b_{1}^{2}\right)^{r}+\cdots \\
& +\left(\begin{array}{c}
2 k \\
2
\end{array}\right) b_{0}^{4}\left(-b_{1}^{2}\right)^{k-1}+b_{0}^{2}\left(-b_{1}^{2}\right)^{k}-b_{0}^{2 k}\left(b_{1}^{2}\right)+\left(\begin{array}{c}
2 k \\
2 k-2
\end{array}\right) b_{0}^{2 k-2}\left(b_{1}^{2}\right)^{2}+\cdots \\
& +\left(\begin{array}{c}
2 k \\
2 k-2 r+2
\end{array}\right) b_{0}^{2 k-2 r+2}\left(-b_{1}^{2}\right)^{r}+\left(\begin{array}{c}
2 k \\
2 k-2 r
\end{array}\right) b_{0}^{2 k-2 r}\left(-b_{1}^{2}\right)^{r+1}+\cdots \\
& +\left(\begin{array}{c}
2 k \\
2
\end{array}\right) b_{0}^{2}\left(-b_{1}^{2}\right)^{k}+\left(-b_{1}^{2}\right)^{k+1}+2\left[\begin{array}{c}
2 k \\
2 k-1
\end{array}\right) b_{0}^{2 k}\left(b_{1}^{2}\right)+\left(\begin{array}{c}
2 k \\
2 k-3
\end{array}\right) b_{0}^{2 k-2}\left(b_{1}^{2}\right)^{2} \\
& +\cdots+\left(\begin{array}{c}
2 k \\
2 k-2 r+1
\end{array}\right) b_{0}^{2 k-2 r+2}\left(-b_{1}^{2}\right)^{r}+\left(\begin{array}{c}
2 k \\
2 k-2 r-1
\end{array}\right) b_{0}^{2 k-2 r}\left(-b_{1}^{2}\right)^{r+1}+\cdots
\end{aligned}
$$




$$
\begin{aligned}
& \left.+\left(\begin{array}{c}
2 k \\
3
\end{array}\right) b_{0}^{4}\left(-b_{1}^{2}\right)^{k-1}+\left(\begin{array}{c}
2 k \\
1
\end{array}\right) b_{0}^{2}\left(-b_{1}^{2}\right)^{k}\right] \\
& =b_{0}^{2 k+2}-\left[\left(\begin{array}{c}
2 k \\
2 k-2
\end{array}\right)+1+2\left(\begin{array}{c}
2 k \\
2 k-1
\end{array}\right)\right] b_{0}^{2 k}\left(b_{1}^{2}\right) \\
& +\left[\left(\begin{array}{c}
2 k \\
2 k-4
\end{array}\right)+\left(\begin{array}{c}
2 k \\
2 k-2
\end{array}\right)+2\left(\begin{array}{c}
2 k \\
2 k-3
\end{array}\right)\right] b_{0}^{2 k-2}\left(b_{1}^{2}\right)^{2}+\cdots \\
& +\left[\left(\begin{array}{c}
2 k \\
2 k-2 r
\end{array}\right)+\left(\begin{array}{c}
2 k \\
k-2 r+2
\end{array}\right)+2\left(\begin{array}{c}
2 k \\
2 k-2 r+1
\end{array}\right)\right] b_{0}^{2 k-2 r+2}\left(-b_{1}^{2}\right)^{r}+\cdots \\
& +\left[\left(\begin{array}{c}
2 k \\
2
\end{array}\right)+1+2\left(\begin{array}{c}
2 k \\
1
\end{array}\right)\right] b_{0}^{2}\left(-b_{1}^{2}\right)^{k}+\left(-b_{1}^{2}\right)^{k+1} \\
& =b_{0}^{2 k+2}-\left(\begin{array}{c}
2 k+2 \\
2 k
\end{array}\right) b_{0}^{2 k}\left(b_{1}^{2}\right)+\left(\begin{array}{c}
2 k+2 \\
2 k-2
\end{array}\right) b_{0}^{2 k-2}\left(b_{1}^{2}\right)^{2}+\cdots \\
& +\left(\begin{array}{c}
2 k+2 \\
2 k-2 r+2
\end{array}\right) b_{0}^{2 k-2 r+2}\left(-b_{1}^{2}\right)^{r}+\cdots+\left(\begin{array}{c}
2 k+2 \\
2
\end{array}\right) b_{0}^{2}\left(-b_{1}^{2}\right)^{k}+\left(-b_{1}^{2}\right)^{k+1} \\
& =\sum_{j=0}^{k+1}\left(\begin{array}{c}
2 k+2 \\
2 k+2-2 j
\end{array}\right) b_{0}^{2 k+2-2 j}\left[-b_{1}^{2}\right]^{j} \\
& \operatorname{Im}\left[b^{2 k+2}\right]=i\left[2 b_{0} b_{1} \sum_{j=0}^{k}\left(\begin{array}{c}
2 k \\
2 k-2 j
\end{array}\right) b_{0}^{2 k-2 j}\left(-b^{2}\right)^{j}\right. \\
& \left.+b_{1}\left(b_{0}^{2}-b_{1}^{2}\right) \sum_{j=0}^{k-1}\left(\begin{array}{c}
2 k \\
2 k-2 j-1
\end{array}\right) b_{0}^{2 k-2 j-1}\left(-b_{1}^{2}\right)^{j}\right] \\
& =b_{1} i\left[2 \sum_{j=0}^{k}\left(\begin{array}{c}
2 k \\
2 k-2 j
\end{array}\right) b_{0}^{2 k-2 j+1}\left(-b_{1}^{2}\right)^{j}+\sum_{j=0}^{k-1}\left(\begin{array}{c}
2 k \\
2 k-2 j-1
\end{array}\right) b_{0}^{2 k-2 j+1}\left(-b_{1}^{2}\right)^{j}\right. \\
& \left.+\sum_{j=0}^{k-1}\left(\begin{array}{c}
2 k \\
2 k-2 j-1
\end{array}\right) b_{0}^{2 k-2 j-1}\left(-b_{1}^{2}\right)^{j+1}\right] \\
& =b_{1} i\left[2 b_{0}^{2 k+1}-2\left(\begin{array}{c}
2 k \\
2 k-2
\end{array}\right) b_{0}^{2 k-1}\left(b_{1}^{2}\right)+2\left(\begin{array}{c}
2 k \\
2 k-4
\end{array}\right) b_{0}^{2 k-3}\left(b_{1}^{2}\right)^{2}+\cdots\right. \\
& +2\left(\begin{array}{c}
2 k \\
2 k-2 r+2
\end{array}\right) b_{0}^{2 k-2 r+3}\left(-b_{1}^{2}\right)^{r-1}+2\left(\begin{array}{c}
2 k \\
2 k-2 r
\end{array}\right) b_{0}^{2 k-2 r+1}\left(-b_{1}^{2}\right)^{r}+\cdots \\
& +2\left(\begin{array}{c}
2 k \\
2
\end{array}\right) b_{0}^{3}\left(-b_{1}^{2}\right)^{k-1}+2 b_{0}\left(-b_{1}^{2}\right)^{k} \\
& +\left(\begin{array}{c}
2 k \\
2 k-1
\end{array}\right) b_{0}^{2 k+1}-\left(\begin{array}{c}
2 k \\
2 k-3
\end{array}\right) b_{0}^{2 k-1}\left(b_{1}^{2}\right)+\left(\begin{array}{c}
2 k \\
2 k-5
\end{array}\right) b_{0}^{2 k-3}\left(b_{1}^{2}\right)^{2}+\cdots \\
& +\left(\begin{array}{c}
2 k \\
2 k-2 r+1
\end{array}\right) b_{0}^{2 k-2 r+3}\left(-b_{1}^{2}\right)^{r-1}+\left(\begin{array}{c}
2 k \\
2 k-2 r-1
\end{array}\right) b_{0}^{2 k-2 r+1}\left(-b_{1}^{2}\right)^{r}+\cdots \\
& +\left(\begin{array}{c}
2 k \\
3
\end{array}\right) b_{0}^{5}\left(-b_{1}^{2}\right)^{k-2}+\left(\begin{array}{c}
2 k \\
1
\end{array}\right) b_{0}^{3}\left(-b_{1}^{2}\right)^{k-1} \\
& -\left(\begin{array}{c}
2 k \\
2 k-1
\end{array}\right) b_{0}^{2 k-1}\left(b_{1}^{2}\right)+\left(\begin{array}{c}
2 k \\
2 k-3
\end{array}\right) b_{0}^{2 k-3}\left(b_{1}^{2}\right)^{2}-\left(\begin{array}{c}
2 k \\
2 k-5
\end{array}\right) b_{0}^{2 k-5}\left(b_{1}^{2}\right)^{3}+\cdots \\
& +\left(\begin{array}{c}
2 k \\
2 k-2 r+1
\end{array}\right) b_{0}^{2 k-2 r+1}\left(-b_{1}^{2}\right)^{r}+\left(\begin{array}{c}
2 k \\
2 k-2 r-1
\end{array}\right) b_{0}^{2 k-2 r-1}\left(-b_{1}^{2}\right)^{r+1}+\cdots \\
& \left.+\left(\begin{array}{c}
2 k \\
3
\end{array}\right) b_{0}^{3}\left(-b_{1}^{2}\right)^{k-1}+\left(\begin{array}{c}
2 k \\
1
\end{array}\right) b_{0}\left(-b_{1}^{2}\right)^{k}\right]
\end{aligned}
$$




$$
\begin{aligned}
= & b_{1} i\left[b_{0}^{2 k+1}\left[2+\left(\begin{array}{c}
2 k \\
2 k-1
\end{array}\right)\right]-b_{0}^{2 k-1}\left(b_{1}^{2}\right)\left[2\left(\begin{array}{c}
2 k \\
2 k-2
\end{array}\right)+\left(\begin{array}{c}
2 k \\
2 k-3
\end{array}\right)+\left(\begin{array}{c}
2 k \\
2 k-1
\end{array}\right)\right]\right. \\
& +b_{0}^{2 k-3}\left(b_{1}^{2}\right)^{2}\left[2\left(\begin{array}{c}
2 k \\
2 k-4
\end{array}\right)+\left(\begin{array}{c}
2 k \\
2 k-5
\end{array}\right)+\left(\begin{array}{c}
2 k \\
2 k-3
\end{array}\right)\right]+\cdots \\
& +b_{0}^{2 k-2 r+1}\left(-b_{1}^{2}\right)^{r}\left[2\left(\begin{array}{c}
2 k \\
2 k-2 r
\end{array}\right)+\left(\begin{array}{c}
2 k \\
2 k-2 r-1
\end{array}\right)+\left(\begin{array}{c}
2 k \\
2 k-2 r+1
\end{array}\right)\right]+\cdots \\
& \left.\left.+b_{0}^{3}\left(-b_{1}^{2}\right)^{k-1}\left[\begin{array}{c}
2 k \\
2
\end{array}\right)+\left(\begin{array}{c}
2 k \\
1
\end{array}\right)+\left(\begin{array}{c}
2 k \\
3
\end{array}\right)\right]+b_{0}\left(-b_{1}^{2}\right)^{k}\left[2+\left(\begin{array}{c}
2 k \\
1
\end{array}\right)\right]\right] \\
= & b_{1} i\left[b_{0}^{2 k+1}\left(\begin{array}{c}
2 k+2 \\
2 k+1
\end{array}\right)-b_{0}^{2 k-1}\left(b_{1}^{2}\right)\left(\begin{array}{c}
2 k+2 \\
2 k-1
\end{array}\right)+b_{0}^{2 k-3}\left(b_{1}^{2}\right)^{2}\left(\begin{array}{c}
2 k+2 \\
2 k-3
\end{array}\right)+\cdots\right. \\
& +b_{0}^{2 k-2 r+1}\left(-b_{1}^{2}\right)^{r}\left(\begin{array}{c}
2 k+2 \\
2 k-2 r+1
\end{array}\right)+\cdots \\
& \left.+b_{0}^{3}\left(-b_{1}^{2}\right)^{k-1}\left(\begin{array}{c}
2 k+2 \\
3
\end{array}\right)+b_{0}\left(-b_{1}^{2}\right)^{k}\left(\begin{array}{c}
2 k+2 \\
1
\end{array}\right)\right] \\
= & b_{1} i \sum_{j=0}^{k}\left(\begin{array}{c}
2 k+2 \\
2 k-2 j+1
\end{array}\right) b_{0}^{2 k-2 j+1}\left(-b_{1}^{2}\right)^{j}
\end{aligned}
$$

By the similar way, we can prove (4).

\section{Results}

We can use (3) and (4) to compute the powers of a quaternion number and the powers of an octonion number.

To compute the power of $a$, replace $b_{0}^{2}$ by $a_{0}^{2}$ and $b_{1}^{2}$ by $a_{1}^{2}+a_{2}^{2}+\cdots+a_{7}^{2}$ in (3) and (4),

$$
\begin{aligned}
& \operatorname{Re}\left[a^{n}\right]=\sum_{j=0}^{n / 2}\left(\begin{array}{c}
n \\
n-2 j
\end{array}\right) a_{0}^{n-2 j}\left[-\left(a_{1}^{2}+\cdots+a_{7}^{2}\right)\right]^{j} \\
& \operatorname{Im}\left[a^{n}\right]=\left(a-a_{0}\right) \sum_{j=0}^{n / 2-1}\left(\begin{array}{c}
n \\
n-2 j-1
\end{array}\right) a_{0}^{n-2 j-1}\left[-\left(a_{1}^{2}+\cdots+a_{7}^{2}\right)\right]^{j} \\
& \operatorname{Re}\left[a^{n}\right]=\sum_{j=0}^{(n-1) / 2}\left(\begin{array}{c}
n \\
n-2 j
\end{array}\right) a_{0}^{n-2 j}\left[-\left(a_{1}^{2}+\cdots+a_{7}^{2}\right)\right]^{j} \\
& \operatorname{Im}\left[a^{n}\right]=\left(a-a_{0}\right) \sum_{j=0}^{(n-1) / 2}\left(\begin{array}{c}
n \\
n-2 j-1
\end{array}\right) a_{0}^{n-2 j-1}\left[-\left(a_{1}^{2}+\cdots+a_{7}^{2}\right)\right]^{j}
\end{aligned}
$$

(7) and (8) give $\left(a_{0}+a_{1} i_{1}+a_{2} i_{2}+\cdots+a_{7} i_{7}\right)^{n}$ when $n$ is an even number and $n$ is an odd number respectively.

Of course the proof is similar to that one we used to prove the powers formulas for a complex number, but to make it clearer, we will write $A$ as:

$$
A=\omega(a)+v(a)
$$

where

$$
\omega(a)=\left[\begin{array}{cccccccc}
a_{0} & -a_{1} & -a_{2} & -a_{3} & -a_{4} & -a_{5} & -a_{6} & -a_{7} \\
a_{1} & a_{0} & 0 & 0 & 0 & 0 & 0 & 0 \\
a_{2} & 0 & a_{0} & 0 & 0 & 0 & 0 & 0 \\
a_{3} & 0 & 0 & a_{0} & 0 & 0 & 0 & 0 \\
a_{4} & 0 & 0 & 0 & a_{0} & 0 & 0 & 0 \\
a_{5} & 0 & 0 & 0 & 0 & a_{0} & 0 & 0 \\
a_{6} & 0 & 0 & 0 & 0 & 0 & a_{0} & 0 \\
a_{7} & 0 & 0 & 0 & 0 & 0 & 0 & a_{0}
\end{array}\right],
$$




$$
v(a)=\left[\begin{array}{cccccccc}
0 & 0 & 0 & 0 & 0 & 0 & 0 & 0 \\
0 & 0 & -a_{3} & a_{2} & -a_{5} & a_{4} & a_{7} & -a_{6} \\
0 & a_{3} & 0 & -a_{1} & -a_{6} & -a_{7} & a_{4} & a_{5} \\
0 & -a_{2} & a_{1} & 0 & -a_{7} & a_{6} & -a_{5} & a_{4} \\
0 & a_{5} & a_{6} & a_{7} & 0 & -a_{1} & -a_{2} & -a_{3} \\
0 & -a_{4} & a_{7} & -a_{6} & a_{1} & 0 & a_{3} & -a_{2} \\
0 & -a_{7} & -a_{4} & a_{5} & a_{2} & -a_{3} & 0 & a_{1} \\
0 & a_{6} & -a_{5} & -a_{4} & a_{3} & a_{2} & -a_{1} & 0
\end{array}\right]
$$

Since $\omega(a)$ will play an important role in our proof, we call it the fundamental matrix. The following proposition presents the main properties of the fundamental matrix $\omega(a)$.

\subsection{Proposition}

Let $a^{\mathrm{T}}=\left[\begin{array}{llllllll}a_{0} & a_{1} & a_{2} & a_{3} & a_{4} & a_{5} & a_{6} & a_{7}\end{array}\right]^{\mathrm{T}}, \bar{a}$ be the conjugate of $a, \lambda$ be a real number, and $I_{8}$ be the identity matrix. Then
(a) $A^{2}=\omega(a) a^{\mathrm{T}}$
(b) $\omega\left(a^{n}\right)=\omega^{n}(a)$
(c) $\omega(\bar{a})=\omega^{\mathrm{T}}(a)$
(d) $\omega(\lambda a)=\lambda \omega(a)$
(e) $\omega(1)=I_{8}$

\section{Proof:}

(a) $a^{2}=[\omega(a)+v(a)] a^{\mathrm{T}}=\omega(a) a^{\mathrm{T}}$, (since $\left.v(a) a^{\mathrm{T}}=0\right)$.

In general $a^{n+1}=a a^{n}, a^{n+1}=\omega(a)\left(a^{n}\right)^{\mathrm{T}}$, ( $n$ is a real number).

(c) $a \bar{a}=\omega(a) \bar{a}^{\mathrm{T}}=a_{0}^{2}+a_{1}^{2}+\cdots+a_{7}^{2}=\|a\|^{2}$

The verification of remaining propositions is straightforward.

Form proposition (a), $a^{2}$ can be computed from:

$$
\begin{aligned}
\omega(a) a^{\mathrm{T}} & =\left[\begin{array}{cccccccc}
a_{0} & -a_{1} & -a_{2} & -a_{3} & -a_{4} & -a_{5} & -a_{6} & -a_{7} \\
a_{1} & a_{0} & 0 & 0 & 0 & 0 & 0 & 0 \\
a_{2} & 0 & a_{0} & 0 & 0 & 0 & 0 & 0 \\
a_{3} & 0 & 0 & a_{0} & 0 & 0 & 0 & 0 \\
a_{4} & 0 & 0 & 0 & a_{0} & 0 & 0 & 0 \\
a_{5} & 0 & 0 & 0 & 0 & a_{0} & 0 & 0 \\
a_{6} & 0 & 0 & 0 & 0 & 0 & a_{0} & 0 \\
a_{7} & 0 & 0 & 0 & 0 & 0 & 0 & a_{0}
\end{array}\right]\left[\begin{array}{c}
a_{0} \\
a_{1} \\
a_{2} \\
a_{3} \\
a_{4} \\
a_{5} \\
a_{6} \\
a_{7}
\end{array}\right] \\
& =\left[\begin{array}{c}
a_{0}^{2}-\left(a_{1}^{2}+\cdots+a_{7}^{2}\right) \\
2 a_{0} a_{1} \\
2 a_{0} a_{2} \\
2 a_{0} a_{3} \\
2 a_{0} a_{4} \\
2 a_{0} a_{5} \\
2 a_{0} a_{6} \\
2 a_{0} a_{7}
\end{array}\right]
\end{aligned}
$$




$$
a^{2}=a_{0}^{2}-\left(a_{1}^{2}+\cdots+a_{7}^{2}\right)+2 a_{0}\left(a_{1} i_{1}+\cdots+a_{7} i_{7}\right)
$$

So, multiplying the first row of $\omega(a)$ by $(a)^{\mathrm{T}}$ gives $\operatorname{Re}\left[a^{2}\right]$, and to obtain $\operatorname{Im}\left[a^{2}\right]$ we just multiply any row (except the first row) of $\omega(a)$ by $a^{\mathrm{T}}$ because the multiplication result will be similar for all other rows, therefore $\operatorname{Im}\left[a^{2}\right]$ will be summation of these results.

In general, since $a^{2 k+2}=a^{2} a^{2 k}$, we will obtain $\operatorname{Re}\left[a^{2 k+2}\right]$ by multiply the first row of $\omega\left(a^{2}\right)$ by $\left(a^{2 k}\right)^{\mathrm{T}}$, and to obtain $\operatorname{Im}\left[a^{2 k+2}\right]$ we multiply any row (except the first row) of $\omega\left(a^{2}\right)$ by $\left(a^{2 k}\right)^{\mathrm{T}}$.

In case $\operatorname{Re}[a]=0$ ( $a$ is a pure octonion) and

$\operatorname{Im}(a)=a_{1} i_{1}+a_{2} i_{2}+a_{3} i_{3}+a_{4} i_{4}+a_{5} i_{5}+a_{6} i_{6}+a_{7} i_{7} \neq 0$, if $n$ is an even number then:

$$
\begin{aligned}
& \operatorname{Re}\left[a^{n}\right]=\left[-\left(a_{1}^{2}+\cdots+a_{7}^{2}\right)\right]^{n / 2} \\
& \operatorname{Im}\left[a^{n}\right]=0
\end{aligned}
$$

And if $n$ is an odd number then:

$$
\begin{aligned}
& \operatorname{Re}\left[a^{n}\right]=0 \\
& \operatorname{Im}\left[a^{n}\right]=a\left[-\left(a_{1}^{2}+\cdots+a_{7}^{2}\right)\right]^{(n-1) / 2}
\end{aligned}
$$

\subsection{Example}

Let $a=i_{2}+2 i_{3}-3 i_{6}+i_{7}$

$$
A=\left[\begin{array}{cccccccc}
0 & 0 & -1 & -2 & 0 & 0 & 3 & -1 \\
0 & 0 & -2 & 1 & 0 & 0 & 1 & 3 \\
1 & 2 & 0 & 0 & 3 & -1 & 0 & 0 \\
2 & -1 & 0 & 0 & -1 & -3 & 0 & 0 \\
0 & 0 & -3 & 1 & 0 & 0 & -1 & -2 \\
0 & 0 & 1 & 3 & 0 & 0 & 2 & -1 \\
-3 & -1 & 0 & 0 & 1 & -2 & 0 & 0 \\
1 & -3 & 0 & 0 & 2 & 1 & 0 & 0
\end{array}\right]
$$

From (10),

$$
\begin{gathered}
\operatorname{Re}\left[a^{6}\right]=[-(15)]^{3}=-3375 \\
\operatorname{Im}\left[a^{6}\right]=0
\end{gathered}
$$

So, $a^{6}=-3375$

$$
A^{6}=\left[\begin{array}{cccccccc}
-3375 & 0 & 0 & 0 & 0 & 0 & 0 & 0 \\
0 & -3375 & 0 & 0 & 0 & 0 & 0 & 0 \\
0 & 0 & -3375 & 0 & 0 & 0 & 0 & 0 \\
0 & 0 & 0 & -3375 & 0 & 0 & 0 & 0 \\
0 & 0 & 0 & 0 & -3375 & 0 & 0 & 0 \\
0 & 0 & 0 & 0 & 0 & -3375 & 0 & 0 \\
0 & 0 & 0 & 0 & 0 & 0 & -3375 & 0 \\
0 & 0 & 0 & 0 & 0 & 0 & 0 & -3375
\end{array}\right]
$$


From (11),

$$
\begin{gathered}
\operatorname{Re}\left[a^{5}\right]=0 \\
\operatorname{Im}\left[a^{5}\right]=\left(i_{2}+2 i_{3}-3 i_{6}+i_{7}\right)[-(15)]^{2}=225\left(i_{2}+2 i_{3}-3 i_{6}+i_{7}\right) \\
A^{5}=\left[\begin{array}{cccccccc}
0 & 0 & -225 & -450 & 0 & 0 & 675 & -225 \\
0 & 0 & -450 & 225 & 0 & 0 & 225 & 675 \\
225 & 450 & 0 & 0 & 675 & -225 & 0 & 0 \\
450 & -225 & 0 & 0 & -225 & -675 & 0 & 0 \\
0 & 0 & -675 & 225 & 0 & 0 & -225 & -450 \\
0 & 0 & 225 & 675 & 0 & 0 & 450 & -225 \\
-675 & -225 & 0 & 0 & 225 & -450 & 0 & 0 \\
225 & -675 & 0 & 0 & 450 & 225 & 0 & 0
\end{array}\right]
\end{gathered}
$$

\subsection{Example}

To compare our formulas with the De Moivre's formula and Euler's formula that were used in [7] to find $a^{7}$ for $a=1+i_{2}+i_{4}+i_{6}$, take

$$
A=\left[\begin{array}{cccccccc}
1 & 0 & -1 & 0 & -1 & 0 & -1 & 0 \\
0 & 1 & 0 & 1 & 0 & 1 & 0 & -1 \\
1 & 0 & 1 & 0 & -1 & 0 & 1 & 0 \\
0 & -1 & 0 & 1 & 0 & 1 & 0 & 1 \\
1 & 0 & 1 & 0 & 1 & 0 & -1 & 0 \\
0 & -1 & 0 & -1 & 0 & 1 & 0 & -1 \\
1 & 0 & -1 & 0 & 1 & 0 & 1 & 0 \\
0 & 1 & 0 & -1 & 0 & 1 & 0 & 1
\end{array}\right]
$$

From (8),

$$
\begin{aligned}
\operatorname{Re}\left[a^{7}\right]=\sum_{j=0}^{3}\left(\begin{array}{c}
7 \\
7-2 j
\end{array}\right)[-(3)]^{j}=1-\left(\begin{array}{l}
7 \\
5
\end{array}\right)(3)+\left(\begin{array}{l}
7 \\
3
\end{array}\right)(9)-\left(\begin{array}{l}
7 \\
1
\end{array}\right)(27)=64 \\
\operatorname{Im}\left[a^{7}\right]=\left(i_{2}+i_{4}+i_{6}\right) \sum_{j=0}^{3}\left(\begin{array}{c}
7 \\
6-2 j
\end{array}\right)[-(3)]^{j} \\
=\left(i_{2}+i_{4}+i_{6}\right)\left[\left(\begin{array}{l}
7 \\
6
\end{array}\right)-\left(\begin{array}{l}
7 \\
4
\end{array}\right)(3)+\left(\begin{array}{l}
7 \\
2
\end{array}\right)(9)-(27)\right] \\
=64\left(i_{2}+i_{4}+i_{6}\right)
\end{aligned}
$$

So, $a^{7}=64\left(1+i_{2}+i_{4}+i_{6}\right)$, and

$$
A^{7}=\left[\begin{array}{cccccccc}
64 & 0 & -64 & 0 & -64 & 0 & -64 & 0 \\
0 & 64 & 0 & 64 & 0 & 64 & 0 & -64 \\
64 & 0 & 64 & 0 & -64 & 0 & 64 & 0 \\
0 & -64 & 0 & 64 & 0 & 64 & 0 & 64 \\
64 & 0 & 64 & 0 & 64 & 0 & -64 & 0 \\
0 & -64 & 0 & -64 & 0 & 64 & 0 & -64 \\
64 & 0 & -64 & 0 & 64 & 0 & 64 & 0 \\
0 & 64 & 0 & -64 & 0 & 64 & 0 & 64
\end{array}\right]
$$


Also let us compute $a^{8}$.

From (7),

$$
\begin{aligned}
& \operatorname{Re}\left[a^{8}\right]=\sum_{j=0}^{4}\left(\begin{array}{c}
8 \\
8-2 j
\end{array}\right)[-(3)]^{j}=1-\left(\begin{array}{l}
8 \\
6
\end{array}\right)(3)+\left(\begin{array}{l}
8 \\
4
\end{array}\right)(9)-\left(\begin{array}{l}
8 \\
2
\end{array}\right)(27)+(81)=-128 \\
& \operatorname{Im}\left[a^{8}\right]=\left(i_{2}+i_{4}+i_{6}\right) \sum_{j=0}^{3}\left(\begin{array}{c}
8 \\
7-2 j
\end{array}\right)[-(3)]^{j} \\
&=\left(i_{2}+i_{4}+i_{6}\right)\left[8-\left(\begin{array}{l}
8 \\
5
\end{array}\right)(3)+\left(\begin{array}{l}
8 \\
3
\end{array}\right)(9)-(8)(27)\right] \\
&=128\left(i_{2}+i_{4}+i_{6}\right)
\end{aligned}
$$

So, $a^{8}=128\left(-1+i_{2}+i_{4}+i_{6}\right)$, and

$$
A^{8}=\left[\begin{array}{cccccccc}
-128 & 0 & -128 & 0 & -128 & 0 & -128 & 0 \\
0 & -128 & 0 & 128 & 0 & 128 & 0 & -128 \\
128 & 0 & -128 & 0 & -128 & 0 & 128 & 0 \\
0 & -128 & 0 & -128 & 0 & 128 & 0 & 128 \\
128 & 0 & 128 & 0 & -128 & 0 & -128 & 0 \\
0 & -128 & 0 & -128 & 0 & -128 & 0 & -128 \\
128 & 0 & -128 & 0 & 128 & 0 & -128 & 0 \\
0 & 128 & 0 & -128 & 0 & 128 & 0 & -128
\end{array}\right]
$$

\section{Conclusion}

The formulas presented in this work are more suitable for computing the powers of an octonion number (the powers of matrices representing an octonion number). These formulas which are derived from binomial expansion also can be used to compute the power of a quaternion number (the powers of matrices representing a quaternion number), and a complex number (the powers of matrices representing a complex number).

\section{Conflicts of Interest}

The author declares no conflicts of interest regarding the publication of this paper.

\section{References}

[1] Dray, T. and Manogue, C.A. (2015) The Geometry of Octonions. World Scientific. https://doi.org/10.1142/8456

[2] Baez, J. (2002) The Octonions. Bulletin of the American Mathematical Society (New Series), 39, 145-205.

[3] Conway, J.H. and Smith, D.A. (2003) On Quaternions and Octonions. Their Geometry, Arithmetic, and Symmetry. A K Peters Ltd., Boca Raton. https://doi.org/10.1201/9781439864180

[4] Graves, R.P. (1882) Life of Sir William Rowan Hamilton. Hodges Figgis, Dublin.

[5] Cayley, A. (1845) On Jacobi's Elliptic Functions, in Reply to the Rev. Brice Bornwin; and on Quaternions. Philosophical Magazine Series, 26, 208-211. 
https://doi.org/10.1080/14786444508645107

[6] Tian, Y. (2000) Matrix Representations of Octonions and Their Applications. Advances in Applied Clifford Algebras, 10, 61. https://doi.org/10.1007/BF03042010

[7] Bektaş, Ö. and Yüce, S. (2019) De Moivre's and Euler's Formulas for the Matrices of Octonions. Proceedings of the National Academy of Sciences, India Section A: Physical Sciences, 89, 113-127. https://doi.org/10.1007/s40010-017-0388-z 